\title{
Mujeres, medio ambiente y desarrollo: \\ ¿Tema de moda, no-tema, tema de la ONU?
}

\author{
Referencia a la situación \\ del debate y del \\ movimiento después de \\ la Conferencia Mundial \\ de la Mujer 1995, \\ celebrada en Pekín*
}

Birte Rodenberg

Universidad de Bielefeld

\section{Resumen}

El conjunto temático "mujeres-medio-ambientedesarrollo" se ha establecido como una discusión independiente en el debate político del desarrollo de los últimos años. El persistente lobbying (cabildeo) delas organizaciones internacionales de mujeres, especialmente antes y durante la Conferencia para el Medio Ambiente y el Desarrollo de las Naciones Unidas en Rio de Janeiro (1992), ha tenido como resultado que actualmente ya no se ponga en duda el

* Original en alemán publicado en Entwicklungsetbnologie (Revista de Ernologra), núm. 1, 1996, traducción: A. Garrido A., Universidad de Bielefeld. 
hecho de que las mujeres del sur son las más afectadas por la degradación global del medio ambiente. Ya entonces era evidente que las representantes del enfoque ecológico feminista eran las críticas más enérgicas de los paradigmas imperantes del desarrollo. Ante todo, prevenían contra el peligro de que las mujeres comprometidas fueran absorbidas por instituciones ecológico-políticas como "amas de casá globales en la protección del medio ambiente ${ }^{\mathrm{n}}$. Sin embargo, en 1995, durante el Foro de las ONG en la Cuarta ConferenciaMundial de la Mujer, celebrada en Pekín, el problema de la ecología ocupaba un lugar marginal en las asambleas principales. El debate en torno a la crisis ecológica y sus repercusiones para las mujeres desaparecía aparentemente- en las discusiones sobre la globalización de la economía y la consiguiente feminización de la pobreza. El artículo versa sobre la historia de las ideas de la discusión, e ilustra la posición actual frente al trasfondo de las conferencias de la ONU que han sido relevantes para la temática en los últimos años.

El debate ecológico de las mujeres en la República Federal de Alemania: de víctima a heroína ecologista

Al cumplirse el décimo aniversario de la catástrofe del reactor atómico de Chernóbil a fines de abril de 1996, también cumplió años el conjunto temático "mujeres, medio ambiente y desarrollo". Actualmente el tema ha vuelto a captar la atención pública en Alemania, "precisamente a tiempo del aniversario del terrible accidente ('GAU')", dirán con amarga ironía aquellas personas que han seguido trabajando y luchando en este ámbito.

Las fluctuaciones en el interés público se deben en gran parte a la falta de arraigo de la política ecológica a nivel medio. El medio ambiente enfermo, por cierto, tiene su cabildeo en las asociaciones nacionales y las redes internacionales, pero no existe un movimiento fuerte e importante que sirva como mediador entre la política comunal local de los afectados, por un lado, y la de los representantes de la política nacional o bien global, por el otro.
Economía, Sociedad y Territorio, vol. 1, núm. 2, 1997, 407-426

Es así como las mujeres con hijos y las embarazadas, con principales afectadas por las precipitaciones radioactivas, fueron protestar a las calles después de la catástrofe nuclear en la primave de 1986. También fuerón ellas, como víctimas, quienes criticar exaltadamente la locura de la factibilidad en la investigación, economía y la política patriarcal. La protesta femenina presental a la ideología destructiva del progreso como uno de los paradigm imperantes de nuestra civilización, y estableć́a una relación con división sexual del trabajo en nuestra sociedad: en su afán $\mathrm{p}$ dominar y comercializar la naturaleza, la sociedad patriarcal asig el trabajo no asalariado de la reproducción a las mujeres, que se vi obligadas - cual si fueran enfermeras - a hacerse cargo del cuidac de los seres humanos que han sufrido daños, y a ocuparse de limpieza -como amas de casa- removiendo los desperdici dispersos en la naturaleza (Gambaroff et al., 1986).

Sin embargo, no fue posible crear un movimiento ecológic feminista amplio y permanente queabordara abiertamente el víncu entre la destrucción de la naturaleza causada por la sociedad y explotación de la fuerza de trabajo femenina. La pequeña ola protestas vehementes, en cambio, desembocó en el así llamac Debute sobre la Renuncia al Consuma (Konsumverzichtsdebatte Éste, por cierto, argumenta frente al contexto económico mundi y también ofrece estrategias concretas de acción para las mujeres d norte; pero los requerimientos fuertemente moralizantes en el deba han irritado a muchas de ellas, de modo que un gran porencial c mujeres ya comprometidas anteriormente, ahora no participan en movimiento.

Aunque en el contexto de la discusión sobre Chernóbil 1 mujeres subrayaron sobre todo su condición de víctimas, ya en es momento también prevenían del peligro de una feminización de responsabilidad. Este acaparamiento de las mujeres de una política c limitación de daños, criticada y llamada posteriormen “domesticación de la protección del medio ambiente" (Wichtericl

' El Debate sobre la Renuncia al Consumo fie introducido en la República Federal Alemania, entre otras, por Maria Mies, quien lo entendía cbmo un 'movimiento de liberaci del consumo". Éste se dirige ante todo a las amas de casa y, en general, a tas mujeres de clase media de los países del norte, a las 'agentes del consumo", instándolas a pres "resistencia con el canasto de compras" (Mies, 1988:294). Se les pide que compren menos, m conscientemente y boicoteando el injusto comerciomundial para colaborar a la formación otra estructura comercial mundial alternativa. A esta incómoda estrategia se le critica q cuestione día tras día nuestros privilegios, que esté pensada como la principal estrategia pa llegar a una sociedad libre de dominación en todo el mundo, y que en vista de la complejid de la situación macroeconómica, la estrategia propuesta abarque tan pocos aspectos. 
1992), fue destacado especialmente por científicas sociales a comienzos de los años noventa, cuando determinados productos textiles alergizantes y el trabajo extra de separar la basura excitaban los ánimos en las bases (Wichterich, 1992; Schultz, 1993).

Pero no es sólo por el hecho de que al estar a cargo de la atención de la familia las mujeres tengan mayor trabajo en el ámbito doméstico con la protección del medio ambiente y los daños ecológicos. También son ellas quienes toman iniciativas a nivel local, organizando grupos de base, de autoayuda, food-coops, etcétera - "naturalmente" sin retribución alguna. Y, como es habitual, siguen sin participar en las decisiones políticas tomadas en las dirigencias de las asociaciones ecologistas. Es así corno en las asociaciones de protección ambiental aproximadamente dos tercios de los socios siguen siendo mujeres. Pero al examinar quiénes ocupan los cargos directivos de las agrupaciones locales, se observa que menos de $20 \%$ son mujeres, como lo demuestra el ejemplo de la Federación Nacional para la Protección de la Naturaleza y el Medio Ambiente de Alemania (Bund für Umwelt- und Naturschutz Deutschland, BUND): las mujeres dirigen sólo $16 \%$ de las agrupaciones locales (Rigos, 1995:40).

Con las diversas acciones de Greenpeace para impedir el planeado hundimiento de la plataforma Brent-Spar durante el verano y otoño de 1995 - que fueron sumamente comentadas en todos los medios-, el tema de las "mujeres y el medio ambiente" volvió a recibir nuevo pábulo: en la prensa las mujeres fueron retratadas como luchadoras comprometidas en el "frente ecológico", como "heroínas ecologistas" (Kamradt, 1995; Klönne, 1995; König, 1995), o como "futura fuerza en la protección del medio ambiente" (Rigos, 1995:40). Pero, al mismo tiempo, constantemente se habla del peligro de que las mujeres sean funcionalizadas para hacer desaparecer los deterioros y la contaminación del medio ambiente: ahora no sólo las activistas se pronuncian categóricamente en favor de la cogestión política. También la prensa trata de evitar la glorificación de las mujeres ecologistas, diciendo que sólo su participación activa en la política ambiental, incluso en puestos de decisión superiores, las protegerá de ser responsabilizadas como “amas de casa ecológicas”.
El debate sobre "mujeres, medio ambiente y desarrollo" desde Rio 1992 hasta Pekín 1995

También en los debates políticos del ámbito internacional, el complejo temático "mujeres y medio ambiente" siempre depende de las fluctuaciones de intereses. El tema fue discutido intensamente en el contexto de la Conferencia sobre el Medio Ambiente y el Desarrollo organizada por las Naciones Unidas (UNCED), que se efectuó en Rio de Janeiro en 1992. En cambio, durante la Cuarta Conferencia Mundial de la Mujer, en Pekín, en septiembre de 1995 la crisis ecológica y sus consecuencias para las mujeres no parecían ser temas importantes de las discusiones políticas.

A continuación referiré algunos de los aspectos esenciales discutidos en el debate internacional sobre lis mujeres, el medio ambiente y el desarrollo. Como en los últimos años las grandes conferencias de las Naciones Unidas han ganado cada vez mayor importancia como plataforma de acción y negociación para el movimiento feminista internacional, expondré los cambios en las posiciones con base en aquellas conferencias de la ONU. Para ello es importante entender estas conferencias no como eventos puntuales, sino como procesos en los cuales los movimientos feministas internacionales tratan de aclarar sus posiciones $\mathrm{y}$ de ejercer influencia tanto en las conferencias preparatorias corno en el foro no gubernamental organizado paralelamenze a la conferencia de la ONU.

Ya en las conferencias mundiales de la mujer efectuadas en la década mundial de la mujer, 1975-1985, proclamada por las Naciones Unidas, se vio claramente que estos foros de las ONG son centros de cristalización de los movimientosfeministas internacionales (Wichterich, 1995:8 y ss). Allí es posible presentar, vincular y fortalecer sus temas, sus posiciones y sus diversos potenciales.' El Foro de las ONG paralelo a la última Conferencia Mundial de la Mujer en Pekín, tuvo gran importancia en este sentido, ya que en él no sólo $\rightsquigarrow$ discutieron diferencias estructurales y aspectos comunes de las diversas realidades en que viven las mujeres en todo el mundo, sino que también se pretendió reducir las líneas de discusión de las cuatro conferencias anteriores a un denominador común para afianzar sus resultados.

${ }^{2}$ Al final ¿e este artículo hay un resumen cronológico de las conferencias. Para mayores detalles y un análisis más extenso de la historia de las conferencias de las Naciones Unidas, véase Wichterich, 1995. 
No fue sólo el hecho de que de los aproximadamente 400 willeres diarios realizados en el recinto del foro, sólo unos 20 (frecuentemente aún menos) estuvieran dedicados al tema del "medio ambiente"; además se vio claramente después de los primeros días de discusiones que no se presentarían nuevos enfoques ni se discutiría intensivamente sobre las estrategias para tratar el problema en el futuro. En vez de ello, los temas centrales de las discusiones pasaron a ser los problemas de la globalización de la economía de mercado, la cogestión política para las mujeres y las violaciones de sus derechos humanos.

Fue casi sintomático para este nuevo enfoque en la discusión internacional, $\mathrm{y}$ además reflejó la pérdida de interés en el tema de crisis ecológica global, el hecho de que -ya en los dos primeros días de trabajo- la WEDO organizara el Segundo Congreso Mundial de la "Mujer por un Planeta Saludable". y que no se ocupara siquiera $50 \%$ de la capacidad de la sala de congresos en aquellos dos días. "E medio ambiente está en receson, fue el comentario desilusionado de Christa Wichterich después de volver del foro:

Tres años después de Rio, el tema está pasado de moda, es un tema secundario - para las mujeres mismas y también para la Naciones Unidas. No es tema en los balances que las Naciones Unidas han presentado en relación a la situación de las mujeres [...] En todas partes la atención está fij en el cambio estructural y en los problemas de th econothe (Wichterich 1996:68).

El hecho de que en el Foro 1995 de las ONG, las asambleas organizadas por la organización política dedicada a problemas ecológicos, la WEDO, resaltaran los problemas económicos de la globalización ${ }^{4}$ y la importancia del poder político de decisión, señala una vez más que la problemática de la ecología es un tema fundamental en todos los ámbitos. Pero también muestra que la crisis ecológica ya no está en el primer lugar de la agenda política de las no directamente afectadas: Además de la feminización de la pobreza, mundialmente, el problema de la violencia y de los

- Con el térnino globalización se designa la ampliación de la economía capitalista de mercado a todo el mundo, lo que implica que las economías nacionales, en determinadas condiciones estructurales (programas de ajuste estructural, por ejemplo), se ven forzadas a participar en el mercado mundial. En este proceso, las instituciones internacionales como las de Bretton Woods y la nueva organización mundial de comercio (World Trade Organization, wro) -además de los consorcios multinacionales- van adquiriendo cada vez mayor poder
e importancia. derechos humanos fue el tema explícito en las discusiones de Pekín. Pero en las reuniones de trabajo donde el tema principal del debate era el medio ambiente, se veía claramente que las mujeres afectadas perseguían una meta pragmática, que es indispensable para asegurar su subsistencia, porque se trataba de "las áreas problemáticas de la alimentación, la salud y el consumo" (Wichterich, 1996:69).

\section{De la ecología feminista a la economía feminista}

El cambio de énfasis en el debate feminista internacional, como se puda observar en el Foro 1995 de las ONG, debe comprenderse como confirmación de la crítica feminista de los paradigmas imperantes del desarrollo, y de la ratificación de las visiones de un modo de vida más sustentable (formuladas anteriormente en el proceso de la UNCED 1991/92), pero con referencia a una economía feminista, no a una ecología feminista.

"El análisis crítico de la creencia básica mortal en el desarrollo" sigue siendo la principal demanda de las grandes organizaciones de mujeres comprometidas del sur, como lo demuestra el enfoque de DAWN; pero el problema de la ecología está integrado en los principios de la crítica feminista de la economía.

En vista de la creciente globalización y feminización de la pobreza, el concepto de las mujeres agrupadas en DAWN se caracteriza por la exigencia de más poder para las mujeres en todos los estratos sociales. Esto se refiere especialmente a su pretensión de lograr acceso a los recursos naturales y control sobre los medios de producción. Para lograr estas metas reclaman -además de mayor poder político de decisión- reformas agrarias a escala global.Esto significa que el debate sobre las mujeres y el medio ambiente ya no es una discusión independiente, sino -concretamente- que el problema de la ecología en el debate político feminista ha pasado a formar parte de la discusión sobre los recursas. Allí los llamados recursos naturales son considerados como recursos sociales, vale decir, no como "un árbol verde y una grulla salvaje" (Bookchin,

${ }^{5} \mathrm{En}$ la red de organización de mujeres DAWN (Development Alternatives with Women for New Era) se ham aerupado mujeres cientificas y activistas del sur (1984) para trabajar y luchar -desde la perspectiva de las mujeres pobres del sur- por un cambio de paradigmas. También fueron ellas quienes en 1985 presentaron el concepto del empowerment ("empoderamiento") en Nairobi. 
- Una mirada retrospectiva al proceso de preparación de la Conferencia Ecológica de las Naciones Unidas, el así llamado Proceso de la UNCED 1991-1992, nos muestra claramente el modo eficaz como - por la influencia que han tenido las mujeres comprometidas- se ha modificado la imagen de la mujer en la política ecologista y del desarrollo.

\section{Retrospectiva: el proceso de la Cumbre de la Tierra}

En la fase de preparación para la confenencia denominada "Cumbre de la Tierra" de las Naciones Unidas, el "escenario de las ONG" vivió un proceso. de autoorganización de las mujeres nunca ames visto. Parque cuando las mujeres (ante todo las norteamericanas) cónstataron que la única alusión a ellas en los borradores del documento de las Naciones Unidas - la Agenda 21- había sido en una parte referente a la "pobreza", fundaron el proyecto Organización de Mujeres para el Medio Ambiente y el Desarrollo (WEDO). ${ }^{3}$ El objetivo de la WEDO era -y la Organización lo ha logrado más allá de la Conferencia de Rio-que las mujeres y su papel central en los problemas ecológicos se hicieran presentes en los documentos de las Naciones Unidas y se ejerciera influencia en el proceso de la UNCED de modo que las demandas de una política ambiental totalmente reformada se rigieran ante todo por las necesidades de las mujeres. La presencia de la WEDO contribuyó a crear y o transmitir una imagen diferente de las mujeres en la discusión feminista sobre el medio ambiente, $\oplus$ decir, que "no sólo deben ser consideradas como víctimas (lo que efectivamente son), sino también como personajes activos con igualdad de derechos en el proceso político" (WEDO, citado en Wichterich, 1992:9). Ias numerosas publicaciones hasta aquel momento no habían presentado a las principales afectadas por la crisis ecológica - las mujeres del sur-, sino sólo como víctimas pasivas, que-como principales proveedoras de agua, leña, alimentos $\mathrm{y}$ forraje de sus unidades domésticas - sufren el.daño inmediato al destruirse estos recursos. Las autoras siempre habían insistido también en su gran potencial de conocimientos y los efectos sustentables e indispensables para la subsistencia de sus relaciones

${ }^{3}$ La WEDO (Women's Environment Developrnent Organization) no sólo es una de las organizaciones ecologistas más grándes de las mujeres, sino que también coordina una de las redes més grandes para proyectos ecológicos y de desarrollo: The Environment and Development Collaborative Web "Daughters of the Earth". con los recursos naturales (Dankelman y Davidson, 1990; Rodda, 1991). En el proceso de la UNCED, sin embargo, se ponía de relieve, ante todo, el aspecto que las presenta como actoras políticas.

En noviembre de 1991 la WEDO organizó el primer Congreso Mundial de la "Mujer por un Planeta Saludable", en Miami; al final de este encuentro se aprobó la Agenda 21 de Acción para las Mujeres. Este documento es un plan de acción visionario y radical, que reclama un cambio ético y civilizatorio de nuestro desarrollo social y económico. En él se exige el empoderamiento (empowerment) de las mujeres junto con "medios de subsistencia sustentables" (sustainablelivelihood). Al optar por este concepto, las mujeres pretendían distanciarse de la consigna del "desarrollo sustentable" (sustainable development) orientada hacia el crecimiento económico.

Las activistas de la WEDO fueron sumamente claras y precisas al declarar que el poder de decisión de las mujeres, como figuras claves en la crisis ecológica, era un requisito indispensable para garantizar la sobrevivencia de tados los seres humanos en el futuro.

Con esto, las activistas no sólo lograron vincular y relacioñar las tres líneas de la discusión -mujeres y medio ambiente, medio ambiente y desarrollo, y mujeres y desartrollo- llevadas siempre bipolarmente, y establecerlas' internacionalmente cotho el debate "Mujeres, Medio Ambiente y Desarrollo" (Women, Environment and Development, WFD), sino que también lograron crear conciencia de que cada tema de dimensiones globales debe ser entendido como tema de las mujeres; valdría decir, que los "temas ,de mujeres" inciden en todos los problemas ecológicos $\mathrm{y}$, ante todo, económicos.

\section{La ecología, ¿tema ausente en Pekín?}

El proceso de la UNCED a principios de los años noventa había actuado de un modo estimulante sobre las activistas ecológicas; en cambio, la Conferencia Mundial de lá Mujer, celebrada en Pekín, no parece haber dado nuevos impulsos al debate ferninista sobre el ambiente. Especialmente las discusiones en el Foro de las ONG causaban la impresión de que la crisis ecológica y sus consecuencias para las mujeres del sur ya no eran un tema de debate en las polémicas feministas internacionales. Las esperanzas de aquéllas, que habían deseado encontrar nuevos conocimientos e *incentivos políticos allí, quedaron defraudadas. 
citudo en Werthof, 1992:141), sino como medios de producción, con low cuales trabajan las mujeres y ganan su sustento. La clave social dedistulbución de estos recursos en este caso pasa a ser una cuestión de poder, que se plantea con respecto a la jerarquía entre los géneros.

En cambio, el aspecto de cómo se manejan los recursos está estrecha y directamente ligado al problema ecológico: ¿se aprovechan de un modo sustentable o se explotan abusivamente con peligro de agotarlos? Lo que se lee en las publicaciones referentes al proceder de las mujeres es, desde hace años, bastante contradictorio. Por un lado, siempre se presenta a las mujeres del sur como sumamente cuidadosas en el manejo de los recursos. Por otro lado, se les acuisa de la destrucción masiva y totalmente desconsiderada de la vegetación y los ecosistemas. En los debates del Foro de las ONG también se dijo claramente que el proceder y comportamiento de las mujeres debe ser considerado en el contexto de su situación social. Esto está asociado con las discusiones científicas en las que se argumenta desde hace tiempo que —n su modo de relacionarse con los recursos naturales - las mujeres, ante todo en los países de África y el sudeste de Asia, siguen un pragmatismo de la supervivencia, una racionalidad especial para actuar (Agarwal, 1986; Lachenmann, 1990). ${ }^{6}$

\section{La discutible cercanía a la naturaleza: el ecofeminismo}

Esta racionalidad de las mujeres para actuar y aprovechar los recursos es un argumento importante en este contexto, que puede atenuar y rebatir las frecuentes inculpaciones en su contra. Pero las defensoras de la posición que considera a las mujeres como expertas pragmáticas de la sobrevivencia también se han expresado en contra de una idealización de las mujeres como particularmente "ligadas a la naturaleza”.

Esta supuesta mayor c e $\mathrm{r}$ d a de las mujeres a la naturaleza, que al parecer las capacita de un modo muy especial para cuidar, proteger y restablecer la naturaleza y el medio ambiente, es un

${ }^{6}$ Especialmente en la discusión sobre $\mathbf{h}$ así llamada "destrucción de recursos dependient de la pobreza", que abarca la crisis de la leña, al igual que el debate sobre el crecimient demográfico motivado por la pobreza, se considera a las mujeres como causantes de la destrucción de la naturaleza. Gran parte de los proyectos para mujeres en el ámbito de la ecología se basan en este punto o bien aún se legitiman con esta posición ideológica (respecto a la crítica, véase Lachenmann, 1990 y 1992). aspecto central del ecofeminismo. Pero si el debate sobre las mujeres, el medio ambiente y el desarrollo, cuyo mayor interés es la problemática del medio ambiente, se considera terminado después del Foro 1995 de Pekín, entonces también ha cambiado la relevancia del enfoque ecofeminista en el debate "mujer y medio ambiente".

En el ecofeminismo, que frecuente y erróneamente equipara con principios de la ecología feminista en general, se hace la diferencia entre el ecofeminismo cultural y el ecofeminismo social. Para el primero es la espiritualidad inherente a la mujer lo que determina su mayor cercanía ala naturaleza. En el segundola mayor vinculación de las mujeres con la naturaleza, contrariamente a los hombres, se atribuye a un proceso sociohistórico de apropiación genérico-específico de corporalidad. El así llamado principiofemenino (Shiva, 1989) tiene carácter social, no biológico? A pesar de este carácter social y de la valorización positiva de la cercanía mayor y diferente de las mujeres a la naturaleza, este enfoque se critica como dicotomizante y esencialista (Agarwal, 1992). El punto central en este debate sobre la construcción de la femineidad ${ }^{8}$ era la equiparación de las mujeres y la naturaleza como víctimas de la violencia y la explotación del sistema social patriarcal capitalista, que predestina a las mujeres de un modo muy especial a ser las "salvadoras" del medio ambiente destruido. Mas, aunque el análisis lógico y filosófico de la estructura triádica de explotación de tantos siglos del conjunto mujeres-naturaleza-pueblos colonizados haya sido y siga siendo importante para encontrar la posición feminista, esta autodefinición de las víctimas como "salvadoras" $\boldsymbol{y}$, por ende, como heroínas ecológicas, podría perjudicar a las mujeres, porque así es fácil transferirles la responsabilidad por el "proceso de curación" de la (madre) Tierra.

Las discusiones en el Foro 1995 de las ONG, sin embargo, han demostrado que - dicho en un tono algo irrespetuoso - ha desaparecido el toque ecofeminista, que aun había determinado los discursos en el proceso de la UNCED. Los llamados apelando a la responsabilidad frente al medio ambiente, como porejemplo "Todas somos hijas de la Tierra", prácticamente ya no se escuchaban, ni en el Congreso Mundial de la "Mujer por un Planeta Saludable"

Para mayores detalles, véanse: Wichterich, 1992:43 y ss.; Mies, 1988; Shiva, 1989; Mies y Shiva, 1995.

3n cuanto a la discusión ecología-mujeres desde el punto de vista de la construcción de femineidad, véanse: Wichterich, 1993; Harcourt, 1994 y Braidotti et al., 1994. 
organizado por la WEDO. Se puede comprobar, en cambio, que la necesidad de hipótesis (eco)filosóficas para explicar el cómo y el por qué del compromiso de las mujeres con relación al medio ambiente está ahora cubierta o bien "aclarada". Las demandas de un nuevo modelo de civilización para el siglo XXI, sin embargo, siguen vigentes, pero el principio femenino tan ponderado en el ecofeminismo, ya no se considera como vehículo estable para lograrlo.

¿Visiones de cambios, pero ninguna estrategia?

Lo que sin duda caracteriza el enfoque ecofeminista y también se refleja en la demanda central de otra ética, la exigencia de un modelo de civilización no basado en el crecimiento económico, es su fuerza visionaria inherente. También en las publicaciones más recientes, que exponen las ideas y la historia del debate del ecofeminismo social, $\boldsymbol{s}$ hace especial hincapié en su actitud crítica desafiante frente a la sociedad y al modelo tradicional de desarrollo (Harcourt, 1994:12; Braidotti et al., 1994:174).

Al cambiar el énfasis en el debate, actualmente también ha pasado a segundo plano la discusión sobre la relación de las mujeres con la naturaleza y el medio ambiente: ya sea la división sexual jerárquica del trabajo, ya sea el principio femenino, el sexo (biológico) o género (social), que inducen a las mujeres a tratar de frenar la destrucción del medio ambiente, en primera línea tiene importancia política la realidad existencial de las mujeres, quienes en general tienen la responsabilidad principal para asegurar la sobrevivencia de la familia y la comunidad.

Bina Agarwal declara que la concepción femenina de la naturaleza y el medio ambiente no es un problema primordial en la problemática global real. Subraya que el argumento que habla en favor de la "mayor cercanía de las mujeres a la naturaleza", puede ser conveniente para algunos movimientos, pero para otros puede ser peligroso. Según ella, la meta no es idealizar nuestra posición como mujeres, sino modificar estructuras existentes de poder (Agarwal, citada en Braidotti et al., 1994:172). Y para organizar este cambio no sólo se requieren visiones y utopías, sino que deben existir, ante todo, estrategias concretas y procesos de negociación para lograrlas.

Empero, casi no se han discutido eventuales estrategias (esa ya fue la crítica más frecuente durante los debates en el Foro de las ONG en Rio de Janeiro), o se han discutido muy poco (Braidotti et

al., 1994:179). Ese debate se había postergado optimistamente al proceso de preparación para la Conferencia Mundial de la Mujer en Pekín, o bien, delegado en el Foro 1995 de las ONG. Pero también en esa oportunidad quedaron frustradas las activistas ecologistas. Este debate tan necesario, aparentemente ha sido transferidocada vez más al proceso de evaluación posterior a las conferencias.

Política internacional ambiental y de desarrollo: las mujeres en peligro de ser funcionalizadas

La "conferencia de las responsabilidades" exigida por muchas personas y casi implorada por la secretaria general de la Conferencia Mundial de la Mujer 1995, señora Gertrude Mongella, tampoco se cumplió en el ámbito "medio ambiente y desarrollo". El lema del "desarrollo sustentable" está presente en el capítulo "Women and Environment" del documento final como una fórmula mágica, sin haber dado cabida a la crítica feminista expresada desde hace años en cuanto a los paradigmas tradicionales del desarrollo.

La "Plataforma de Acción" de Pekín, en cambio, da fe con palabras elocuentes acerca de cuánto se necesita y aprecia a las mujeres como expertas para manejar la crisis e incluso como recurso para superar la crisis ambiental. En el documento no sólo se introdujo el concepto de "empoderamiento" para mujeres, reinvindicado por las activistas de la WEDO en el proceso de la UNCED 1991/92, sino que este concepto también llegó a ser el elemento estructural central en el proceso ambiental y de desarrollo. Esto, por cierto, no necesariamente se debe celebrar como éxito, ya que las instituciones nacionales e internacionales de desarrollo lograron así un doble triunfo: porque la demanda de mayor poder político para las mujeres en realidad estaba pensada como respuesta a la funcionalización de las mujeres como redentoras de la crisis.

Cabe mencionar como aspecto favorable que el compromiso continuado y tenaz de las mujeres - no sólo desde el proceso de la UNCED - ha repercutido en algunos puntos, y que su crítica ha conducido a mucho más que un cambio simplemente cosmético. Pero no ha variado en cuanto a la concepción de las Naciones Unidas de que existe compatibilidad entre crecimiento económico y sustentabilidad, entre economía de mercado y protección de los recursos. Para las Naciones Unidas, para sus actores nacionales e 
internacionales, sigue vigente un paradigma de crecimiento que lo sumo - quizás sea retocado eficazmente. ${ }^{9}$

También en el ámbito de los proyectos políticos de desarrollo y fomento a la mujer, de tipo ecológico, persiste el peligro -después de la Conferencia de Pekín- de que las mujeres puedan ser fácilmente funcionalizadas; eso significa que la "promoción de la mujer" -como lo formula Lachenmann para África- "debe ser considerada como un intento manifiesto de integrar a las mujeres renitentes definitivamente a la economía del crecimiento" (Lachenmann, 1992:202). Mientras la "instrumentalización de la importancia económica de la mujer en el modelo de mercado existente, orientado en el crecimiento económico" (Dederichs-Bain, 1995:14), siga siendo la meta abierta y encubierta de todos los "esfuerzos de desarrollo", también existirá la tendencia de funcionalizar el compromiso femenino en el área ecológica para un crecimiento sustentable (mejor aún: ipermanente!) -ya sea en el ámbito de la política nacional o internacional de promoción de la mujer, o bien, en el de los proyectos ecológicos por las ONG (Rodenberg, 1994).

No obstante, no pretendo hablar en favor de la dualidad "bueno-malo", porque una confrontación entre las mujeres del sur organizadas en varias ONG y las Naciones Unidas, las instituciones de Bretton Woods y los/las políticos/as estatales del desarrollo, por el otro lado, ya no es pertinente. Las discusiones en el Foro 1995 de las ONG han manifestado claramente que la política cle esas organizaciones ya nose caracteriza solamente por visiones radicales y aspiraciones de autonomía mediante compromisos a pequeña escala. El objetivo de sus reivindicaciones es más bien llegar a tener mayor poder de decisión - también en las mesas de conferencias y negociaciones de la "gran política" - y más participación en las principales instituciones y organizaciones.

Ese es el debate por las "nuevas alianzas" que en los Últimos años se ha llevado cada vez con mayor intensidad entre las ONG dedicadas a la ecología (Bebbington y Farrington, 1993:123 y ss.). También en el contexto del debate feminista ecologista se hacen y se aprueban las reflexiones acerca del "cómon y "con quién" se

- En la plataforma de acción jurídico-internacional no obligatoria, se menciona principalmente a los gobiernos. Se les pide redistribuir los medios financieros existentes para poder comenzar con los cambios recomendados. La exigencia de medios adicionales nuevos siempre se menciona solamente 'en el contexto de otras fuentes de financiamiento posible, ante todo el sector privado" (Dederichs-Bain, 1995:13). Precisamente la carencia de financimiento de la plataforma es lo que confiere un carácter más bien retórico a sus temas. ampliará la cooperación -no sólo entre las diversas ONG, sino también mucho más alfá (Braidotti et al., 1994:176 y ss.). Peró ên todas partes existen voces, autque no suficientes, que previenen del peligro de ser funcionalizadas: Irene Santiago, por ejemplo, miembro del comité de organización del Foro 1995 de las ONG, usa la metáfora del río y su pequeño afluente, qu al confluir modifican su forma, pero solamente porque el río más pequeño és absorbido por el más grande. Y termina con la advertencia de que "las instituciones no ceden el poder; es preciso que las mujeres huchen por él”. 
I. Conferencia Mundial de la Mujer y Foro ONG: 1975 en la Ciudad México

II. Conferencia Mundial de la Mujer y Foro ONG: 1980 en Copenhague

m. Conferencia Mundial de la Mujer y Foro ONG: 1985 en Nairobi

IV. Conferencia Mundial de la Mujer y Foro ONG: 1995 en Pekín/Huairou

\section{Glosario:}

DAWN . Development Alternatives with Women for a New Era (Organización de mujeres científicas y activistas del sur)

Foro ONG Foro de las Organizaciones No Gubernamentales que se organiza paralelamente a una conferencia de las Naciones Unidas

UNCED UN Conference on Environment and Development (Conferencia de las Naciones Unidas sobre Medio Ambiente y Desarrollo) en Rio de Janeiro, 1992

UNEP UN Environment Program (Programa de Medio Ambiente de las Naciones Unidas)

WED. Women and Environment in Development (Mujeres

Conferencias de política del desarrollo de las Naciones Unidas y foros ONG

Conferencia UNEP preparatoria de la UNCED: 1991 en Miami

Primer Congreso Mundial de la Mujer: "Mujeres por un Planeta Saludable", organizado por la WEDO: 1991 en Miami

Conferencia de la ONU sobre el Medio Ambiente y el Desarrollo (UNCED): 1992 en Rio de Janeiro

Conferencia de la ONU sobre los Derechos Humanos: 1993 en Viena

Conferencia de la ONU sobre Población y Desarrollo: 1994 en El Cairo

Conferencia Cumbre Social de la ONU: 1995 en

Copenhague

Segundo Congreso Mundial de la Mujer: "Mujeres por un Planeta Saludable", organizado por la WEDO dentro

del Foro de las ONG con motivo de la N Conferencia Mundial de la Mujer: 1995 en Huairou/Pekín

Women's Environiment Development Organization (Organización de mujeres ecologistas para el desarrollo), fundada en 1991 durante el proceso de preparación de la Conferencia las Naciones Unidas en Rio de Janeiro

Bibliografía

Agarwal, Bina (1986), Cold Hearth and Barren Slopes. The Woodfuel Crisis in the Third World, Nueva Delhi, Zed Books.

- (1992), "The Gender and Environment Debate: Lessons from India", Feminist Studies, vol. 18, núm. 1, pp. 119-159.

Bebbington, Anthony y John Farrington et d. (1993), Reluctant Partners? Non-governmental Organizations, the State and Sustainable Agricultural Development, Londres y Nueva York, Routledge.

Braidotti, Rosi et al. (1994), Women, the Environment and Sustainable Development. Towards a Theoretical Synthesis, Londres y Nueva Jersey, Zed Books.

Dankelmann, Joan e Irene Davidson (1990), Frauen und Umwelt in den südlichen Kontinenten, Wuppertal, Lamuv. 
DAWN (Development Alternatives with Women for a New Era) (1995), Securing our Grains and Moving Forward to the 21st Century, documento inédito para la N Conferencia Mundial de la -Mujer en Pekín, septiembre, Barbados.

Dederichs-Bain, Birgit (1995), IV. Weltfrauenkonferenz 1995: Bericht über die Arbeit in der deutschen Delegation aus Sich akkreditierter NRO-Vertreterinnen, Informe inédito, Bonn.

Gambaroff, Marina et al. (1986), Tschernobyl bat unser Leben verändert. Vom Ausștieg der Frauen, Reinbek, Rowohlt.

Harcourt, Wendy (1994), "Negotiating Positions in the Sustainable Development Debates Situating the Feminist Perspective", en W. Hartcourt (ed:), Feminist Perspectives on Sustainable Development, Londres y Nueva Jersey, Sed Books.

Kamradt, Barbara (1995), “Wieviele Frauen steuern mit?”, Emma, núm 5, Documentos “Öko-Heldinnen”, pp. 64-66.

Klönne, Gisa (1995), "Mein Männerbund", Emma, núm. 5, Documentos “Öko-Heldinnen", p. 63.

König, Uta (1995), "Schwester David", Emma, núm. 5, Documentos “Öko-Heldinnen”, pp. 58-62.

Lachenmann, Gudrun (1990), Ökologische Krise und sozialer Wandel in afrikanischen Ländern, Saarbrücken, Breitenbach.

(1992), "Von der Unsichtbarkeit zur Verletzlichkeit zur Pflichtorganisation der Frauen. Widersprüche in der Strukturanpassungs- und Frauenpolitik in Afrika", en Rolf Hofmeier, Rainer Tetzlaff y Regina Wegemund (eds.), Afrika - Überleben in einer ökologisch gefäbrdeten Umwelt (escrito en VAD núm. 14, pp. 194-208), Münster, Hamburg, LrT-Verlang.

Mies, Maria (1988), Patriarchat und Kapital. Frauen in der internationalen Arbeitsteilung, Berlín, Rotpunkt.

y Vandana Shiva (1995), Ökofeminismus. Beiträge aus Theorie und Praxis, Zurich, Rotpunkt.
Quistorp, Eva-Maria (ed.) (1993), Frauen, Umwelt, Entwicklung 1007 Frauenprojekte. Die Grünen im Exropaparlament, Bonn.

Rigos, Alexandra (1995), “Umweltverbände: Lippenbekenntnisseaus der Chefetagew ${ }^{w}$ Natur, núm. 9, p. 40.

Rodda, Annabel (ed.) (1991), Women and the Environment, Londres y Nueva Jersey, Zed Books.

Rodenberg, Birte (1994), "Mehr als Überlebenspragmatismus - Zur Handlungsrationalität, von Frauen in der Ökologiebewegung", Entwicklangsetbnologie, vol. 3, núm. 2, pp. 5665.

(1995), "Visionen von Veränderung, aber keine Strategien? Frauen, Weltwirtschaft und Entwicklung",Frauensolidarität, núm. 54, pp. 7-8.

(1996), "Frauen, Umwelt und Entwicklung: Modethema, Non-Thema, UN-Thema?Zum Stand des Diskurses und der Bewegung nach der Weltfrauenkonferenz 1995 in Peking”, Entwicklungsetbnologie, vol. 5, núm.1, pp. 25-42.

Schultz, Irmgard (ed.) (1993), Globalhaushalt. Globalisierung von Stoffströmen - Feminisierung von Verantwortung, Frankfurt/M., Verlag für interkulturelle Kommunikation.

Shiva, Vandana (1989), Das Geschlecht des Lebens: Frauen, Ökologie und Dritte Welt, Berlín, Rotbuch.

United Nations (1995), Report $\&$ the Fourth World Conference on Women. Beijing Declaration and Platform for Action, Nueva York, ONU

WEDO (Women's Environment Development Organization) (1995), Daughters of the Earth (programa de la conferencia), Nueva York, WEDO.

Werlhof, Claudia von (1992), "Zum Natur- und Gesellschaftsbegriff im Kapitalismus", en Veronika Bennholdt-Thomsen, Maria Mies, y Claudia von Werlhof, Frauen, die Letzte Kolonie, Berlín, Rotpunkt, tercera edición. 
Wichterich, Christa (1992), Die Erde bemuttern. Frauen und Ökologie nach dem Erdgipfel in Rio, Colonia, Fundación Heinrich Böll, e.V.

- (1993), “Die Rückkehr der weisen Frauen”, Peripherie, núms. 51/52, pp. 120-136.

- (1995), Frauen der Welt Vom Fortschritt der Ungleichbeit, Gottingen, Lamuv.

- (1996), Wir sind das Wunder, durcb das wir überleben. Die 4. Weltfrauenkonferenz in Peking, Colonia, Fundación Heinrich Böll, e.V.
Especificacionespara la entrega de los artículos de la revista Economia, Sociedad y Territorio.

$$
3 \cdots
$$

La entrega de un trabajo para su publicación en la revista Economía, Sociedad y Territorio implica para el colaborador el compromiso de no someterlo a otras publicaciones en español al momento de su aprobación para su aparición dentro de lb revista. La publicaciónadmite textos en otros idiomas encargandose ésta de la traducción al español. En los casos en los cuales sean enviados en español se anexará un ejemplar en el idioma original.

Se sugiere que los artículos tengari una extensión de 15 cuartillas como mínimo y 40 como máximo, incluidas gráficas, cuadros, notas y bibliografía; las reseñas podrán tener entre 5 y 10 cuartillas; cada número de la revista esta compuesto de 280 cuartillas como máximo de extensión (incluyendo cuadros, gráficas y todo material gráfico que se incluya en el número).

La presentación de los textos deberá enmarcarse dentro de los siguientes lineamientos:

1.-Los artículos deberán de presentarse en su versión final y completas ya que no se admitiran cambios iniciado el proceso de produreción de la publicación.

$$
\text { : }
$$

2.-Los textos se presentarán impresos en matriz de puntos o en laser a doble espacio $(1,5)$ en tipo curier o times de 11 puntos; los textos que sean entregados mecanografiados deberán estar a doble espacio en cuartillas de 28 líneas y aproximadamente 64 golpes por línea sobre papel grueso; por ningún motivo serán recibidos sobre papel copia y/ o copias al carbón. A estos materiales se le sumará el artículo en procesador de palabras, de preferencia en un disquette de 3.5 y en Word perfect 5.1, Word 6 o almacenarse bajo código de TEXTO DOS (ASCII).

3.-Las referencias bibliográficas se harán en el texto, entre paréntesis, de acuerdo al siguiente modelo: apellido del autor(a), año de edición, número de la página.

Las notas a pie de página, los cuadros y las gráficas se agruparán al final del artículos después de la bibliografía. Las gráficas y cuadros deberán presentarse impresos en originales claros y precisos que 
permitan su publicación; en el caso de ser enviados en disquette deberán de estar almacenados en Excel 5 de ninguna manera serán recibidos en otro programa o versiones posteriores a ésta.

En el texto deberá de indicarse la colocación aproximadamente de estos materiales gráficos.

La lista de referencias bibliográficas deberan presentarse completas y en el siguiente orden: apellido y nombre del autor (a) año de edición, nombre del libro, país o ciudad, editorial. ejemplo:

Legorreta, Jorge (1992), "Expansión urbana de la ciudad de México" en A. Bassols, J. Delgadillo y F. Torres (comps.), El desarrollo regional en México: Teoría y práctica, México, IIE, UNAM.

4.--La página inicial de cada artículo deberá contener: a) resumen en español con extensión máxima de 200 palabras.; b) nombre del autor (a), su filiación institucional, grados académicos, área de interés principal y dos referencias bibliográficas de su autoría; c) número telefónico (fax), dirección electrónica (E-mail) y dirección postal para su eventual localización.

5.-Los artículos y su manejo son competencia del coordinador responsable del número al cual los autores tendrán que dirigirse para cualquier duda o aclaración.

\section{El Colegio Mexiquense, A.C.}
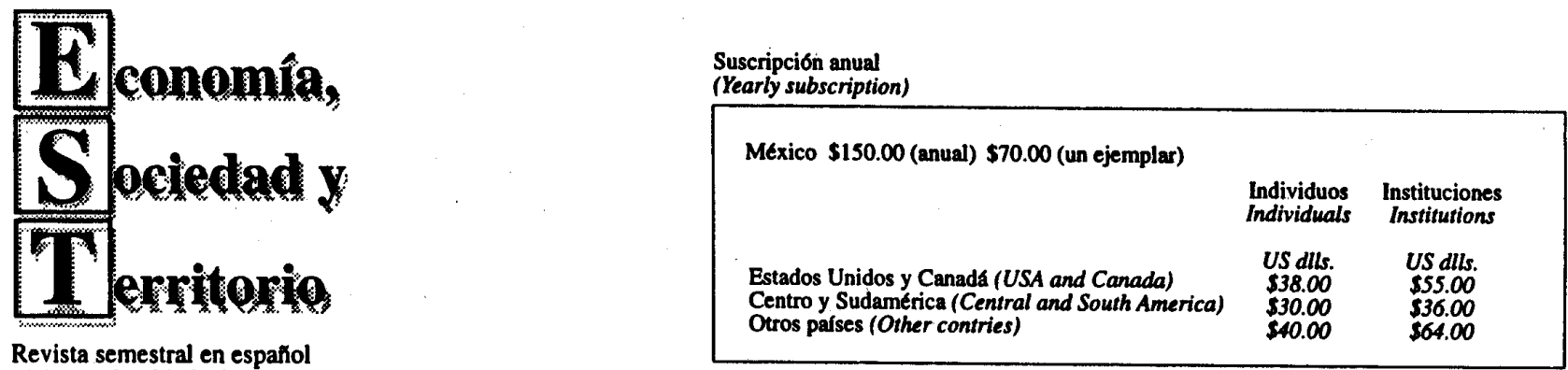

Revista semestral en español

(A journal published twiece a year in Spanish)

Adjunto cheque o giro bancario a nombre de El Colegio Mexiquense, A.C., núm. (Enclosed bank check or order payable fo)
Banco

Enviar a: El Colegio Mexiquense, A.C.

(Send 1o) Departamento de adquisiciones y libreria.

Apartado postal 48-D

Toluca 50120 , México

México

Nombre

Dirección

$\begin{array}{ll}\text { (Name) } & \text { Direccion } \\ \text { (Address) }\end{array}$

CP

Ciudad _ _ Estado

(Zip code) (Ciry) (State)

Pars

Teléfono y fax

(Telephone and) 